\title{
Cardiovascular manifestations of HIV infection: A review.
}

\author{
Dhiraj Kumar*, Varun Deokate \\ King Edward Memorial Hospital, Mumbai, Maharashtra, India
}

\begin{abstract}
In recent times with increased number of patients suffering from HIV all around the world and with progressively increasing life span with HAART (highly active retroviral therapy). Concomitantly there has been an increase in cardiovascular diseases. This review highlights the complex interaction between HIV infection, atherosclerosis, left ventricular dysfunction, pericardial disease, pulmonary hypertension etc. The relationship between the HAART and dyslipidemia and coronary artery disease.
\end{abstract}

Keywords: HAART, Pulmonary hypertension, Atherosclerosis.

\section{Introduction}

The clinical spectrum of HIV disease in humans is as vast and diverse as its implications in the current scenario of path breaking improvements in the science of medicine. As of 2016, 36.7 million (30.8 million-42.9 million) people globally were living with HIV [1]. 20.9 million People were accessing antiretroviral therapy in June 2017 [1]. 1.8 million (1.6 million-2.1 million) people became newly infected with HIV in 2016 [1]. 1 million (8, 30, 000-1.2 million) people died from AIDS-related illnesses in 2016 (Figure 1) [1].

Cardiac abnormalities associated with HIV infection are diverse, encompassing atherosclerotic CAD to malignancy (Kaposi's sarcoma). In this review, we will try to cover the pathophysiology, diagnosis, complications and treatment modalities of each of these cardiovascular manifestations of HIV.

\section{Atherosclerosis}

Accelerated atherosclerosis occurs in persons infected with $\mathrm{HIV}$, irrespective of the presence or absence of traditional coronary risk factors. When compared with the general population affected patients are of younger age and have single vessel disease, with plaque rupture implicated as the cause of myocardial infarction [2].

Endothelial dysfunction resulting from increased expression of adhesion molecules such as ICAM-1, E-selectin and inflammatory cytokines such as TNF- $\alpha$, IL-6 and vWF is the most plausible link between HIV infection and atherosclerosis [3].

Antiretroviral therapy (ART) has significantly modified the history of human immunodeficiency virus (HIV) infection and is responsible for delaying virus replication and preserving the CD4+T cell count. Although the importance of ART in the treatment of HIV cannot be underestimated, some of the classes of drugs used in ART regimens, especially protease inhibitors have detrimental effects on the lipid and glucose metabolism in the body. Thus owing to the intrinsic atherosclerotic effects associated with the infection itself and ironically, the metabolic derangements associated with ART, CAD/CVD has become one of the most common cause of mortality and morbidity in PLHIV and the most challenging to treat.
PIs are known to have high affinity for the catalytic site of the HIV protease enzyme and thus bind and inhibit the homologous protein that is involved in lipid metabolism (Figure 2). A 12-amino acid sequence that comprises the catalytic region of the HIV protease has $63 \%$ homology to the region that incorporates a lipid-binding domain related to the lowdensity lipoprotein receptor (LRP) and 58\% homology to a C-terminus of the cytoplasmic retinoic acid-binding protein type 1 (CRABP-1) [4]. CRABP-1 is a ubiquitous protein that binds all retinoic acid (AR) intracellularly and exhibits retinoic acid for cytochrome P450 3A (CYP3A), which is responsible for converting this substrate into cis-9-retinoic acid [4]. In turn, cis-9-retinoic acid is the only ligand to the retinoid $\mathrm{X}$ receptor (RXR), which has heterodimeric function with the peroxisome proliferator-activated receptor gamma (PPAR- $\gamma$ ) in adipocyte nuclei. When binding to RXR or PPAR- $\gamma$ occurs, apoptosis and upregulation of adipocyte differentiation and proliferation are inhibited, with PPAR- $\gamma$ being more functional in subcutaneous than central adipose tissue. PIs appear to bind to the homologous region within CRABP-1 and thus inhibit the binding of retinoic acid [4]. This process leads to the reduction in the production of cis-9- retinoic acid, with consequent reduction of RXR activity, and finally, it leads to the decrease of the differentiation and increase of apoptosis of peripheral adipocytes. Another PI action site is centered on the protein-related LRP. LRP is an important hepatic receptor for the removal of chylomicrons in the postprandial period. LRP is also coexpressed in the capillary endothelium with lipoprotein lipase (LPL). The LPL-LRP complex cleaves triglycerides circulating in free fatty acids and glycerol for fat storage, mainly in the central region of the body, which characterizes the lipodystrophic syndrome [4].

Protease inhibitors such as amprenavir and fosamprenavir, with or without boost ritonavir or lopinavir with ritonavir have the strongest association with MI. The NRTIs, abacavir and didanosine have also been associated with MI. NNRTIs (nevirapine and efavirenz), entry inhibitors and integrase inhibitors do not appear to increase the risk of MI (Figure 3) [5,6].

Thus, statins can be used in dyslipidemic patients with HIV, as long as there is no drug interaction (Figure 4). Recent studies have demonstrated that rosuvastatin is more effective than other statins in doing so. Treatment of ischaemic heart disease in 


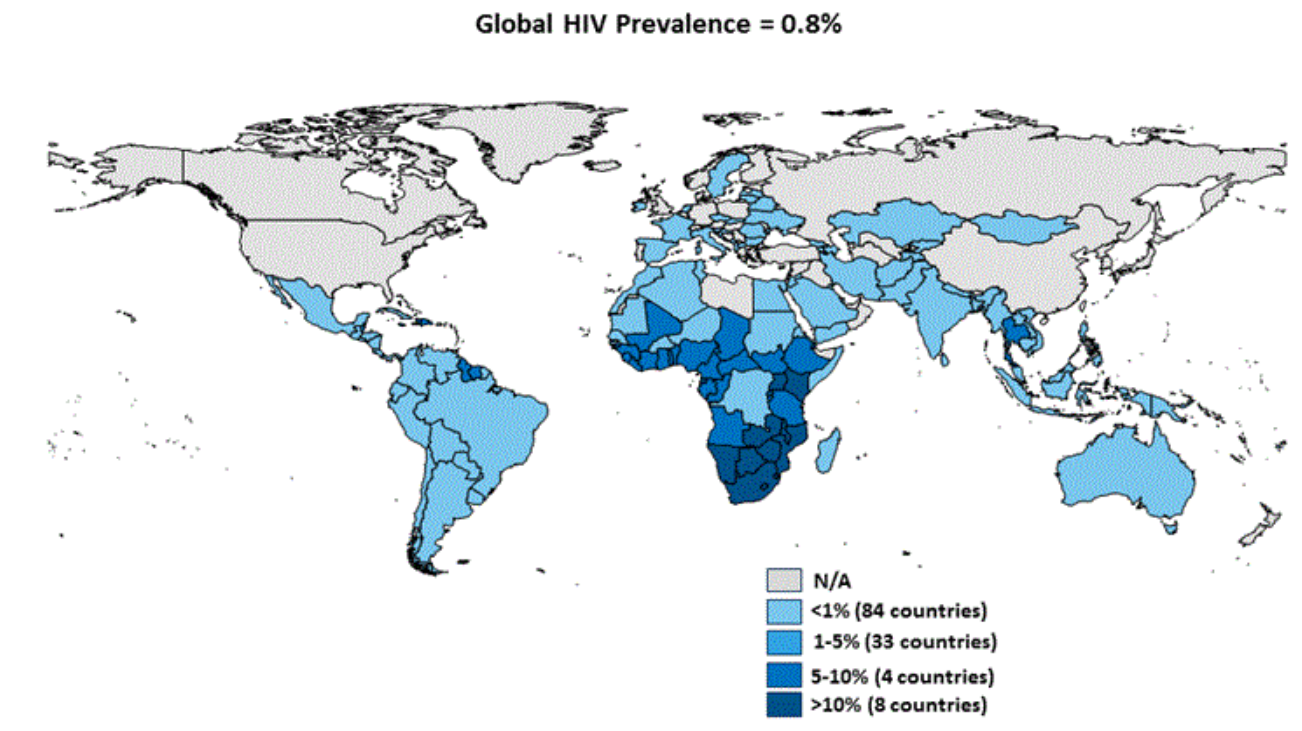

NOTES: Data are estimates. Prevalence includes adults ages 15-49.

SOURCES: Kaiser Family Foundation, based on UNAIDS, AIDSinfo, Accessed July 2017

Figure 1. Adult HIV prevalence 2016.

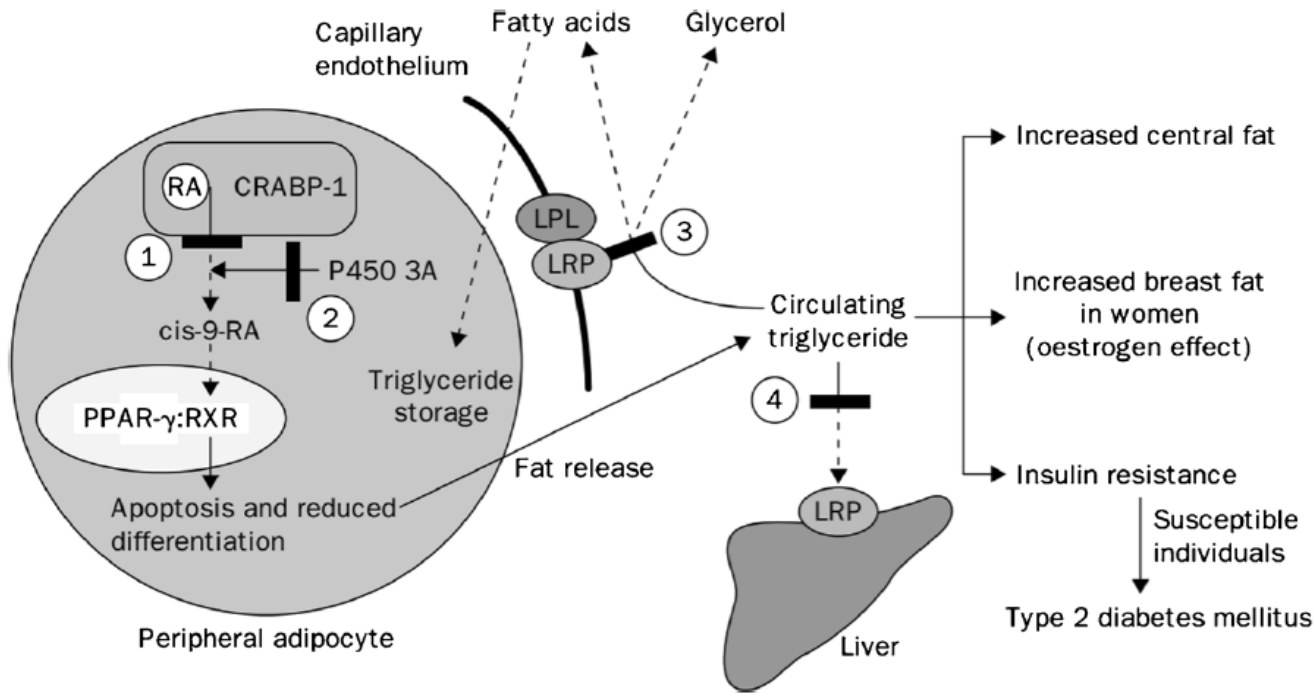

Figure 2. The PI action sites are numbered and circled. RA, retinoic acid; CRABP-1, cytoplasmic retinoic acid-binding protein type 1; PPAR- $\gamma$, peroxisome proliferator-activated receptor gamma; $R X R$, retinoid $X$ receptor; LRP, low-density lipoprotein receptor; LPL, lipoprotein lipase.

PLWHIV is the same as the standard guideline based therapy for ischaemic heart disease in non HIV infected persons.

\section{Left ventricular dysfunction}

In the pre-HAART era, the 2 to 5 year incidence of symptomatic heart failure ranged from 4 to $28 \%$ [7]. In this era, heart failure was often expressed as symptomatic, systolic LV dysfuntion with LV dilatation and was almost exclusively seen in patients with advanced HIV disease (AIDS). Even in the current era of HAART, this type of clinical picture has persisted in those geographical areas where HAART has not reached. The incidence of this type of HIV-associated cardiomyopathy has decreased dramatically from the pre-ART era, from 25.6 cases per 1000 person years to 3.9 cases, according to one recent review [8]. Even in the HAART era, echocardiographic abnormalities related to the cardiac structure have persisted (18\% have LV systolic dysfunction, $6.5 \%$ have $\mathrm{LVH}$ and $40 \%$ have left atrial dilatation), but the diagnosis of heart failure includes many more asymptomatic PLWHIV and often refers only to systolic or diastolic dysfunction detected by echocardiography [9].

Patients with HIV who have LV systolic dysfunction can be asymptomatic or can have NYHA III or IV symptoms with heart failure. Anemia, concurrent pulmonary infections, pulmonary hypertension, malnutrition and malignancy can confound the clinical picture. Higher viral loads and lower CD4 counts were associated with heart failure in PLWHIV. Rapid onset heart failure has a grim prognosis in all HIV infected patients with more than half dying of cardiac failure within 12 months [10].

The pathophysiology of LV dysfunction in PLWHIV is 


\begin{tabular}{|c|c|c|c|c|}
\hline \multicolumn{5}{|c|}{ Currently Recommended First-Line Agents are in Bold } \\
\hline Antiretroviral & Total Cholesterol & LDL-C & HDL-C & Triglycerides \\
\hline \multicolumn{5}{|l|}{ PIs (boosted) } \\
\hline Lopinavir & $\uparrow \uparrow$ & $\uparrow \uparrow$ & $\leftrightarrow / \downarrow$ & $\uparrow \uparrow \uparrow$ \\
\hline Atazanavir & $\uparrow$ & $\leftrightarrow / \uparrow$ & $\leftrightarrow / \downarrow$ & $\leftrightarrow$ \\
\hline Fosamprenavir & $\uparrow$ & $\uparrow$ & $\leftrightarrow / \downarrow$ & $\uparrow \uparrow$ \\
\hline Saquinavir & $\uparrow \uparrow$ & $\uparrow \uparrow$ & $\leftrightarrow / \downarrow$ & $\uparrow$ \\
\hline Darunavir & $\uparrow$ & $\uparrow$ & $\leftrightarrow / \downarrow$ & $\uparrow$ \\
\hline Tipranavir & $\uparrow \uparrow$ & $\uparrow \uparrow$ & $\leftrightarrow / \downarrow$ & $\uparrow \uparrow \uparrow$ \\
\hline \multicolumn{5}{|l|}{ NNRTIs } \\
\hline Efavirenz & $\uparrow$ & $\uparrow$ & $\uparrow$ & $\uparrow$ \\
\hline Nevirapine & $\uparrow$ & $\uparrow$ & $\uparrow \uparrow$ & $\leftrightarrow / \uparrow$ \\
\hline NRTIs & & & & $\leftrightarrow / \uparrow$ \\
\hline Tenofovir & $\leftrightarrow / \uparrow$ & $\leftrightarrow / \uparrow$ & $\leftrightarrow / \uparrow$ & \\
\hline Abacavir & $\leftrightarrow / \uparrow$ & $\uparrow$ & $\uparrow$ & $\uparrow$ \\
\hline Lamivudine & $\leftrightarrow$ & $\leftrightarrow$ & $\leftrightarrow$ & $\leftrightarrow$ \\
\hline Zidovudine & $\uparrow$ & $\uparrow$ & $\uparrow$ & $\uparrow \uparrow$ \\
\hline Stavudine & $\uparrow \uparrow$ & $\uparrow \uparrow$ & $\uparrow$ & $\uparrow \uparrow$ \\
\hline CCR5 Inhibitors & & & & \\
\hline Maraviroc & $\leftrightarrow$ & $\leftrightarrow$ & $\leftrightarrow / \uparrow$ & $\leftrightarrow$ \\
\hline Integrase Inhibitors & & & & \\
\hline Raltegravir & $\leftrightarrow / \uparrow$ & $\leftrightarrow / \uparrow$ & $\leftrightarrow / \uparrow$ & $\leftrightarrow$ \\
\hline
\end{tabular}

Figure 3. Effects of HIV drugs on lipid metabolism [6].

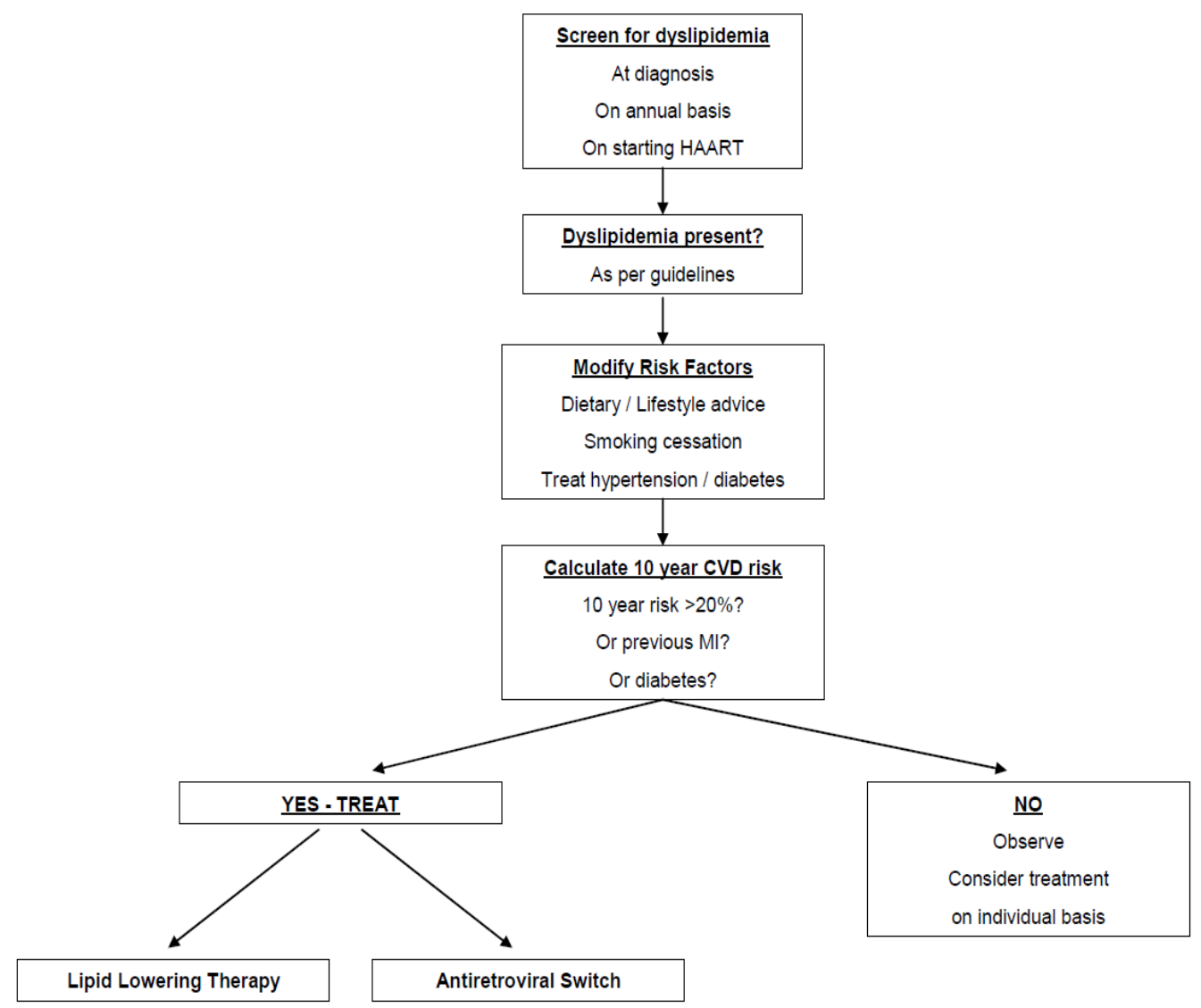

Figure 4. Algorithm for management of HIV associated dyslipidemia [6].

attributable to several factors such as MI, the HIV infection itself (tissue damage from myocarditis, effects of viral proteins), opportunistic infections, autoimmune responses to viral infection, nutritional deficiencies and cytokine overexpression (TNF- $\alpha$, TGF- $\beta$, endothelin-1 and NO) [11]. Nutritional deficiencies are common in PLWHIV. Of importance is selenium deficiency, which is a reversible cause of cardiomyopathy
(Keshan disease). Selenium deficiency also increases the virulence of coxsackievirus on cardiac tissue. LV hypertrophy is more common in HIV-infected patients than in controls. After adjustment for age and traditional risk factors, HIV patients were 2.4 times more likely to have diastolic dysfunction than controls. Overall, 50\% of PLWHIV had diastolic dysfunction compared with $29 \%$ of uninfected controls [12] (Figure 5). 
Therapy for dilated cardiomyopathy associated with HIV is similar to that for non-ischaemic cardiomyopathy. Thus, angiotensin-converting enzyme inhibitors, b-blockers, and aldosterone antagonists should be used, although trials of these drugs have not been done in PLWHIV with heart failure. ART is not specific therapy for heart failure; however, the incidence of heart failure declined dramatically after the introduction of ART. Whether ART can reverse heart failure caused by an established cardiomyopathy is not known. On the other hand, ART drugs such as AZT have direct myocardial toxicity, and ART may accelerate coronary atherosclerosis, ultimately leading to LV dysfunction. As of now, the best strategy is to prevent heart failure with HAART [12].

Heart transplantation with excellent long-term survival has been reported in small numbers of HIV infected patients. The fear that immunosuppression in such patients might lead to AIDS has proved unfounded, and the notion that HIV infection should be a contraindication to cardiac transplantation is no longer tenable [13].

After medical therapy is begun, 4 monthly echocardiography follow up is advised. If function continues to worsen even after 2 weeks of medical therapy, cardiac catheterization and endomyocardial biopsy should be considered [14].

\section{Pulmonary hypertension}

The first case of human immunodeficiency virus-associated pulmonaryarterial hypertension (HIV-PAH) was reported in 1987, in an HIV-infected patient who died of PAH and glomerulonephritis. Since that time PAH has become a well- recognized complication of HIV infection. Modern treatment with antiretroviral therapy has improved survival for patients with HIV infection so that cardiovascular disease and other non-acquired immunodeficiency syndrome (AIDS) conditions are increasingly encountered as these patients live longer. PAH occurs in approximately $0.5 \%$ of PLWHIV [14].

Most patients with HIV infection do not develop PAH, and it remains unclear how HIV leads to the development of PAH in some HIV-infected patients. HIV has never been shown to directly infect pulmonary vascular endothelial cells, but HIV viral antigens are present in the pulmonary endothelium and may directly stimulate abnormal apoptosis, growth, and proliferation. Certain genetic mutations in HIV viral antigens (nef) may predispose to the development of HIV-PAH $[15,16]$. Bone morphogenic protein receptor 2 (BMPR-2) mutations, associated with familial $\mathrm{PAH}$, result in decreased signalling through BMPR-2(16). The HIV-1 that protein (transcriptional transactivator) represses BMPR-2 gene expression in human macrophages in vitro, interfering with BMP-BMPR-2 transcriptional regulation [17].

Current guidelines recommend that all HIV-infected patients be treated with antiretroviral agents regardless of CD4 T-cell count and viral load. Therefore, all patients with HIV-PAH should, if possible, be treated with antiretroviral therapy. It remains unclear, however, if antiviral therapy in and of itself is useful to treat PAH in patients with HIV - PAH. Beneficial effects of phosphodiesterase inhibitors in patients with HIV-PAH have been reported in small series and case reports [18]. The endothelin receptor antagonist bosentan has been specifically
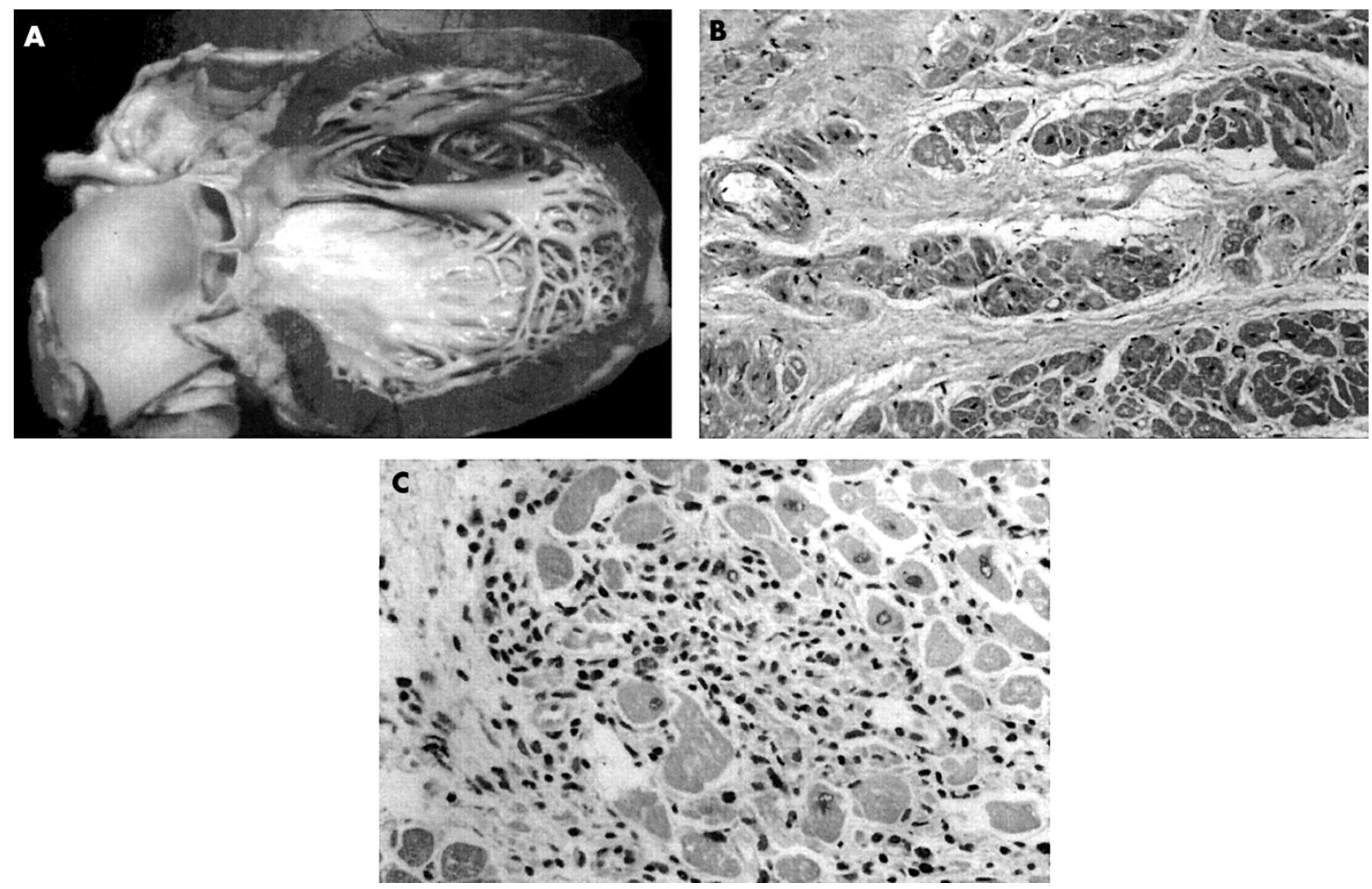

Figure 5. HIV related cardiomyopathy. (A) The heart is enlarged, principally from ventricular dilatation, and there is mild hypertrophy with diffuse endocardial fibrous thickening. Histological examination (B) may reveal myocyte hypertrophy with increased interstitial collagen or (C) evidence of myocarditis with lymphocytic infiltrate and myocyte necrosis [13]. 
studied in patients with HIV-PAH. The BREATHE-4 trial was a prospective study of 16 HIV-PAH patients treated with bosentan for 16 weeks. NYHA classification was improved by at least 1 class in 14 patients, cardiac index improved by $39 \%$, mean pulmonary arterial pressure decreased by $21 \%$, and 6 MWD improved by $91+/-60$ meters. Ambrisentan has not been studied specifically in patients with HIV-PAH, but it does not have the same potential drug interactions with antiretroviral medications, which makes it simpler to use in patients with HIV-PAH. Improvements in hemodynamics and exercise tolerance have been observed in small series of patients treated with intravenous and subcutaneous prostanoids [19].

\section{Pericardial effusion}

In the pre -HAART era, pericardial effusion occurred in up to $11 \%$ of patients with AIDS, the incidence of which has decreased with the initiation of HAART as demonstrated by a recent study [20].

Effusions are generally small and asymptomatic. Lower CD4 counts predispose the patients to pericardial effusions. HIV should always be suspected in a patient with unexplained pericardial effusion or tamponade (Figure 6).

The effusion is often a part of the generalised serous-effusive process that also involves the pleural and peritoneal spaces. This is due to the capillary leak syndrome due to increased cytokine production in patients with HIV infection (usually in AIDS patients). Other most common cause is concurrent TB infection, which in itself may directly cause pericardial effusion and sometimes tamponade. Other less common causes include uremic pericarditis, bacterial pericarditis, other opportunistic infections and malignancy.

Diagnostic workup should be done in all the cases to provide specific therapy as per the etiology. Cases of tamponade should undergo urgent pericardiocentesis. Regular (at least 1 monthly or sooner if clinically indicated) echocardiography follow-up is advised for cases of asymptomatic pericardial effusion [14].

\section{Endocarditis}

The incidence of infective endocarditis in PLWHIV is the same as compared to people without HIV and similar risk behavior [14]. The autoimmune response to bacterial endocarditis is implicated in the valvar destruction associated with endocarditis. Thus, even though salmonella endocarditis is common in PLWHIV as a consequence of increased incidence of salmonella bacteremia (due to immune compromise), they respond to antibiotics and are less likely to sustain valvar damage due to a paucity of immune response [14]. Other common organisms associated with infective endocarditis include staphylococcus aureus, fungal endocarditis such as aspergillus fumigatus, candida species and Cryptococcus neoformans [14].

Fulminant course of infective endocarditis have a grim prognosis in PLWHIV. Operative indications include hemodynamic compromise, persistent bacteremia despite intravenous antibiotics to which the organism is sensitive, persistent embolization and severe valvar destruction, in a patient with reasonable life expectancy after surgery [14].

Marantic endocarditis or non-bacterial thrombotic endocarditis (NBTE), the preferred term, describes a condition in which friable, fibrinous clumps of platelets and red cells affect the cardiac valves. Unlike other forms of endocarditis, these lesions are not thought to be infective in origin, and show no evidence of an inflammatory reaction and little cellular organization. NBTE is difficult to identify at post mortem but had been found in over $1 \%$ of HIV-negative individuals depending on the particular group under scrutiny [21].

\section{Malignancy}

Malignancy affects PLWHIV, mostly later in the disease course. Kaposi sarcoma is a low grade malignancy derived from



Figure 6. Pericardial effusion in PLAX view in a patient with HIV. 
mesenchymal or endothelial cells. It is the most common AIDS related neoplasm. It is associated with HHV 8 infection and affects upto $35 \%$ of AIDS patients, mostly homosexuals and the incidence is inversely related to the CD4 count. At autopsy, heart involvement is observed with $5-8 \%$ of patients with cutaneous and visceral forms of Kaposi sarcoma. Rarely the heart is the sole site of involvement. Clinical signs of the disease are scarce and non-specific. It can be suspected in patients with pericardial effusions associated with an epicardial location of a tumor. Pericardial fluid in patients with cardiac Kaposi sarcoma is serosanguinous, without malignant cells or infection. Kaposi sarcoma is difficult to treat, and most die of opportunistic infections related to advanced immunosuppression (Figure 7) [22].

AIDS-related lymphomas are high-grade B-cell tumors, consisting of large-cell immunoblastic or small noncleaved-cell (Burkitt's or Burkitt-like) lymphomas in approximately 80-90\% of cases. Typically the AIDS lymphomas present in extra nodal sites, such as the central nervous system, gastrointestinal tract and bone marrow. Malignant lymphoma presenting as a primary cardiac tumor is rare; however, its incidence is apparently increasing in $\mathrm{HIV}+$ patients. Clinical presentation of cardiac lymphomas, either primitive or secondary, is varied. Symptoms range from dyspnea to chest pain. Many patients have intractable congestive heart failure, arrhythmias or pericardial effusions, but others do not show clinical evidence of cardiac involvement, at least in the initial phase of the disease. Clinical suspicion of heart involvement by malignant lymphoma should be confirmed by endomyocardial biopsy [22].

\section{Long $Q T$ interval}

HIV infection is associated with QT prolongation and torsades de pointes ventricular tachycardia and the incidence increases with progression to AIDS. The risk for QT prolongation was $16 \%$ with HIV alone and 30\% with both HIV and HCV infection $[14,23,24]$.

\section{Autonomic dysfunction}

Early clinical signs of autonomic dysfunction in PLWHIV include syncope, presyncope, diminished sweating, diarrhoea, bowel/ bladder dysfunction and impotence. In one study, heart rate variability, Valsalva ratio, cold pressor testing, hemodynamic responses to isometric exercises, tilt table testing and standing showed that autonomic dysfunction occurred in patients with HIV infection and more so with AIDS [25].

\section{Conclusion}

To conclude, routine systemic cardiac evaluation, including comprehensive history and cardiac examination, are essential for HIV infected persons. The history should include traditional risk factors, previous opportunistic infections, environmental exposures, and therapeutic and illicit drug use.
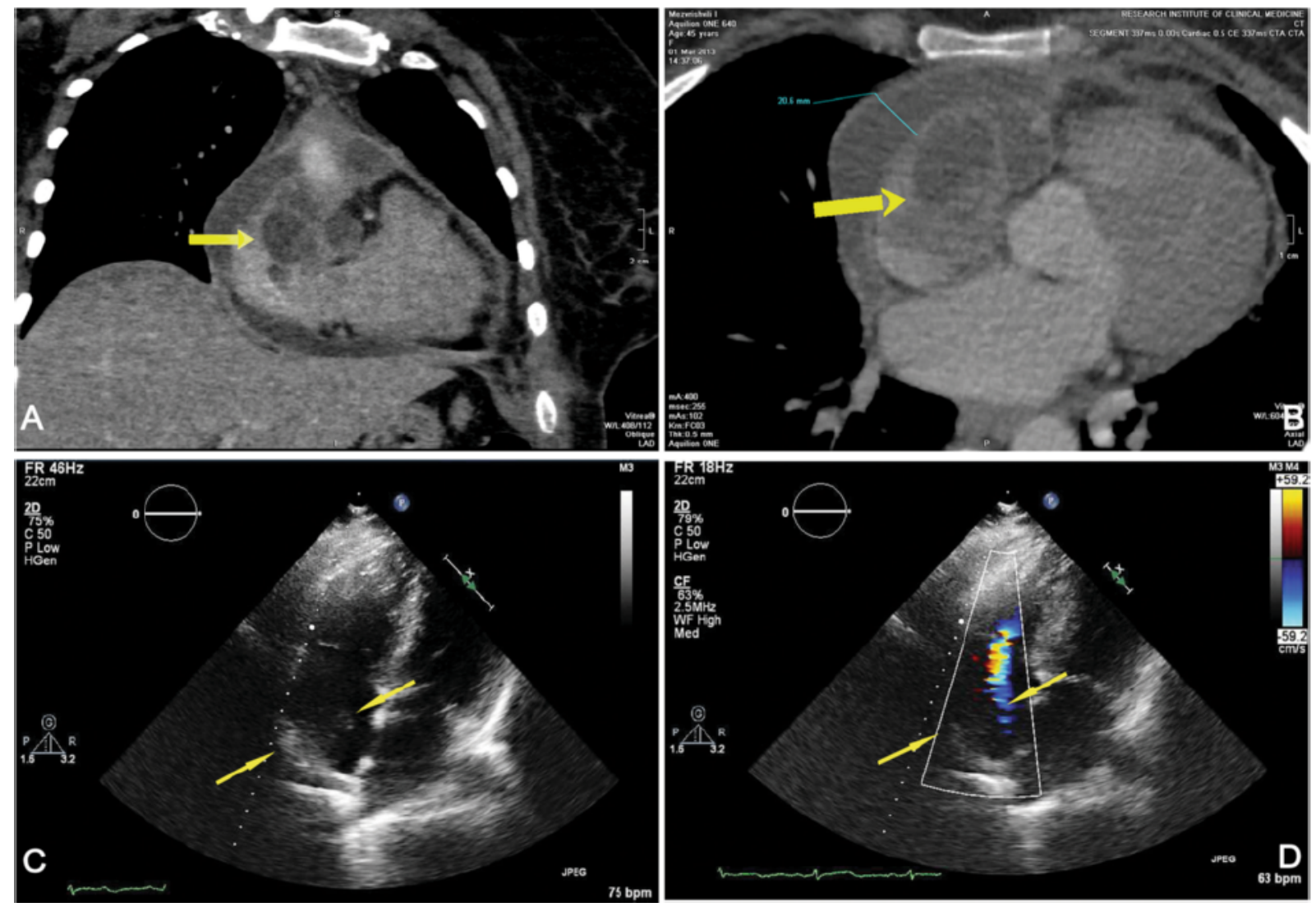

Figure 7. Kaposi sarcoma of heart. a, b Computed Tomographic (CT) coronary angiography. Low-density tumorous mass (50 mm in diameter) at the level of auricle of the right atrium. $c, d$ Transesophageal echocardiography. Tumor in the auricle of the right atrium, spreading toward superior vena cava [23]. 
Asymptomatic HIV-related cardiac disease can be fatal, and cardiac symptoms, when present, are often disguised by the secondary effects of HIV infection, so systematic echocardiographic monitoring is warranted. Primarily, HAART remains the mainstay of treatment of cardiovascular involvement in HIV.

\section{References}

1. http://www.unaids.org/en/resources/fact-sheet citation

2. Longenecker CT, Hoit BD. Imaging atherosclerosis in HIV: carotid intima-media thickness and beyond. Translational Research. 2012;159:127-39.

3. DAD Study Group. Class of antiretroviral drugs and the risk of myocardial infarction. New England J Medicine. 2007;356:1723-35.

4. Carr A, Samaras K, Chisholm DJ, et al. Pathogenesis of HIV-1-protease inhibitor-associated peripheral lipodystrophy, hyperlipidaemia, and insulin resistance. The Lancet. 1998;351:1881-3.

5. Lang S, Mary-Krause M, Cotte L, et al. Impact of individual antiretroviral drugs on the risk of myocardial infarction in human immunodeficiency virus-infected patients: a casecontrol study nested within the French Hospital Database on HIV ANRS cohort $\mathrm{CO}_{4}$. Archives of internal medicine. 2010;170:1228-38.

6. Feeney ER, Mallon PW. HIV and HAART-Associated Dyslipidemia. The Open Cardiovascular Medicine Journal. 2011;5:49-63.

7. Lipshultz SE, Easley KA, Orav EJ, et al. Left ventricular structure and function in children infected with human immunodeficiency virus: the prospective P2C2 HIV multicenter study. Circulation. 1998;97:1246-56.

8. Remick J, Georgiopoulou V, Marti C, et al. Heart failure in patients with human immunodeficiency virus infection: epidemiology, pathophysiology, treatment, and future research. Circulation. 2014;129:1781-9.

9. Mondy KE, Gottdiener J, Overton ET, et al. High prevalence of echocardiographic abnormalities among HIV-infected persons in the era of highly active antiretroviral therapy. Clinical Infectious Diseases. 2011;52:378-86.

10. Currie PF, Jacob AJ, Foreman AR, et al. Heart muscle disease related to HIV infection: prognostic implications. BMJ. 1994;309:1605-7.

11. Yearley JH, Mansfield KG, Carville AA, et al. Antigenic stimulation in the simian model of HIV infection yields dilated cardiomyopathy through effects of TNF $\alpha$. Aids. 2008;22:585-94.

12. Hsue PY, Hunt PW, Ho JE, et al. Impact of HIV infection on diastolic function and left ventricular mass. Circ Heart Fail. 2010;3:132-9.

13. Prendergast BD. HIV and cardiovascular medicine. Heart. 2003;89:793-800.
14. Zipes DP. Braunwald's heart disease: a textbook of cardiovascular medicine. BMH Medical Journal-ISSN 2348-392X. 2018 Mar 2.

15. Almodovar S, Knight R, Allshouse AA, et al. Human Immunodeficiency Virus nef signature sequences are associated with pulmonary hypertension. AIDS Res Hum Retroviruses. 2012;28:607-18.

16. Caldwell RL, Gadipatti R, Lane KB, et al. HIV-1 TAT represses transcription of the bone morphogenic protein receptor-2 in U937 monocytic cells. J Leukoc Biol. 2006;79:192-201.

17. Leong $\mathrm{MH}$, Farber HW. Noninfectious pulmonary complications of HIV. Clin Pulm Med. 2006;13:194-202.

18. Schumacher YO, Zdebik A, Huonker M, et al. Sildenafil in HIV-related pulmonary hypertension. AIDS. 2001;15:1747-8.

19. Gillies H, Wang X, Staehr P, et al. PAH therapy in HIV: lack of drug-drug interaction between ambrisentan and ritonavir. Am J Respir Crit Care Med. 2011;183:A5913.

20. Lind A, Reinsch N, Neuhaus K, et al. Pericardial effusion of HIV-infected patients-results of a prospective multicenter cohort study in the era of antiretroviral therapy. Eur J Medical Research. 2011;16:480.

21. Currie PF, Sutherland GR, Jacob AJ, et al. A review of endocarditis in acquired immunodeficiency syndrome and human immunodeficiency virus infection. European Heart Journal. 1995;16:15-8.

22. D'amati G, Gioia CR, Gallo P. Pathological Findings of HIV $\square$ Associated Cardiovascular Disease. Annals of the New York Academy of Sciences. 2001;946:23-45.

23. Makharoblidze E, Goishvili N, Mchedlishvili M, et al. Primary Kaposi's sarcoma of the heart in nonimmunodeficient patient: case report and literature review. Diagnostic pathology. 2015;10:111.

24. Sani MU, Okeahialam BN. QTc interval prolongation in patients with HIV and AIDS. Journal of the National Medical Association. 2005;97:1657.

25. Correia D, DE RESENDE RO, PERTILLI LA, et al. Power Spectral Analysis of Heart Rate Variability in HIV Infected and AIDS Patients. Pacing and Clinical Electrophysiology. 2006;29:53-8.

\section{*Correspondence to:}

Dhiraj Kumar

King Edward Memorial Hospital

Mumbai

Maharashtra

India

E-mail: dhiraj1645@gmail.com 